\title{
Ossification of the cervical posterior longitudinal ligament: a review
}

\author{
NANCY EPSTEIN, M.D. \\ Department of Neurological Surgery, The Albert Einstein College of Medicine, Bronx; and \\ Department of Neurosurgery, The North Shore-Long Island Jewish Health System, Manhasset and \\ New Hyde Park, New York
}

\begin{abstract}
Ossification of the cervical posterior longitudinal ligament (OPLL) represents a continuum beginning with hypertrophy of the posterior longitudinal ligament (PLL) followed by progressive coalescence of centers of chondrification and ossification. Early OPLL mimicking disc disease appears opposite multiple disc spaces associated with significant retrovertebral extension, helping to differentiate it from spondylosis. On computerized tomography examinations, the single- and double-layer signs indicate possible dural penetration with the increased potential for an intraoperative cerebrospinal fluid fistula during dissection. Direct ventral resection of OPLL in patients younger than 65 years of age is optimal and includes single- or multilevel anterior corpectomy with fusion, the latter accompanied by posterior fusion. For patients older than the age of 65 years, with a well-preserved cervical lordosis, laminectomy with or without fusion and/or laminoplasty may suffice in providing indirect dorsal decompression. Patients undergoing circumferential procedures with halo devices are managed with a specific anesthetic protocol, including awake intubation and positioning with intraoperative monitoring of somatosensory evoked potentials, electromyography, and the option of undergoing motor evoked potential monitoring. Intubation is maintained during the 1st postoperative night. When circumferential procedures are performed intubation is always maintained during the 1st postoperative night, and fiberoptic postoperative extubation is electively performed by specifically trained anesthesiologists when deemed appropriate. Patients exhibiting three or more major risk factors are considered candidates for delayed extubation and rarely, tracheostomy. Repeated anterior surgery, operations lasting more than 10 hours, involving four or more levels (including C-2), obesity, asthma, and blood transfusions of more than $4 \mathrm{U}(1000-1200 \mathrm{ml})$ are all considered major risk factors.
\end{abstract}

\section{KEY WORDS • cervical spine • ossification • posterior longitudinal ligament • spinal surgery}

\section{COMPOSITION OF THE POSTERIOR LONGITUDINAL LIGAMENT}

Collagen and elastin fibers are densely concentrated centrally in the PLL, beginning at the clivus and extending to the sacrum. This ligament is widest at the disc spaces and narrowest at the mid-vertebral levels. It is approximately 1 to $2 \mathrm{~mm}$ thick centrally and thins out laterally. Initial hypertrophy of the PLL due to fibroblastic hyperplasia is followed by increased collagen deposition. Progressive mineralization and cartilaginous ingrowth

Abbreviations used in this paper: $\mathrm{ACF}=$ anterior corpectomy with fusion; $\mathrm{ADF}=$ anterior discectomy with fusion; $\mathrm{CSF}=$ cerebrospinal fluid; $\mathrm{CT}=$ computerized tomography; DISH $=$ diffuse idiopathic skeletal hyperostosis; Gd-DTPA = gadolinium-diethylenetriamine pentaacetic acid; HLA = human leukocyte antigen; $\mathrm{JOA}=$ Japanese Orthopaedic Association; MEP = motor evoked potential; $\mathrm{MR}=$ magnetic resonance; OALL $=$ ossification of the anterior longitudinal ligament; OPLL $=$ ossification of the posterior longitudinal ligament; PLL = posterior longitudinal ligament; $\mathrm{PWF}=$ posterior wiring and fusion; $\mathrm{SSEP}=$ somatosensory evoked potential; $\mathrm{VB}=$ vertebral body; $2 \mathrm{D}=$ two-dimensional; $3 \mathrm{D}=$ threedimensional. form ossification centers that eventually mature into haversian canals.

Osteogenicity of the PLL has been well-documented in both Japan and the United States, the latter in non-Asian patients. ${ }^{38,47,50,55}$ In Japan, immunohistochemical evaluation of PLL cells obtained from anterior cervical surgery revealed "up-regulation of proliferating cell nuclear antigen." ${ }^{65}$ In the United States Epstein and colleagues ${ }^{6,38}$ showed that osteocalcin synthesis occurs in patients with radiographically confirmed OPLL while confirming its absence in spondylotic tissues.

\section{GENETICS}

A genetic locus for OPLL is most likely located close to the HLA site on chromosome 6-p. ${ }^{54,55}$ Patients with DISH, half of whom have OPLL, test positive for HLA antigen. ${ }^{69}$ An autosomal-dominant mode of OPLL inheritance is frequently inferred as one quarter of the siblings of OPLL patients manifesting the disease and demonstrating two concurrent strands of HLA. ${ }^{59,70,74}$ Other genetically modulated factors, including increased concentrations of growth hormone receptors, activins, and the bone morphogenetic proteins, BamHI 10/10 kb, and HindIII 19/19 $\mathrm{kb}$ were also found in OPLL patients. ${ }^{47,54,55,78}$ 


\section{CLASSIFICATION OF OPLL}

One quarter of North Americans and Japanese patients with cervical myelopathy exhibit OPLL. . $2,30,35,73$ Seventy percent of the time OPLL is found in C2-4 and $15 \%$ is found in T1-4, and $15 \%$ in L1-3. Neural injury occurring in the presence of OPLL stems from direct mechanical or indirect ischemic compromise. Cervical OPLL also appears twice as often in males as in females. ${ }^{11,30,31,72}$

\section{MYELOPATHY SCALES}

Two myelopathy grading scales are used: the Nurick Scale and the JOA Scale. The Nurick Myelopathy Scale includes: Grade 0, intact, mild radiculopathy without myelopathy; Grade I, mild myelopathy; Grade II, mild-tomoderate myelopathy; Grade III, moderate myelopathy; Grade IV, moderate-to-severe myelopathy; and Grade V, severe myelopathy, quadriplegia. ${ }^{11-14,20,22,25,30,31,35}$ The JOA Scale catalogues the severity of myelopathy by using a 17point scale. ${ }^{68,80,81}$ Yononbu, et al., ${ }^{79}$ recently investigated and confirmed the reliability of the JOA scoring system.

\section{CLINICAL PRESENTATION OF OPLL}

Patients with early OPLL typically present in their mid40 s with radiculopathy or mild myelopathy. Those with more mature OPLL usually become symptomatic in their mid-50s with moderate-to-severe myelopathy. Although symptoms are usually gradual in onset, $10 \%$ may precipitously develop myelopathy after mild trauma, and many new deficits may prove irreversible despite surgical decompression. ${ }^{43,50}$

\section{NEUROIMAGING OF OPLL}

\section{Plain X-Ray Films}

Absolute cervical stenosis (canal diameter $<10 \mathrm{~mm}$ ) and relative cervical stenosis (canal diameter 10-13 mm) predispose patients with OPLL to developing more severe deficits earlier in the clinical course. When the "occupancy ratio" is greater than $40 \%$ as defined by the thickness of OPLL divided by the canal diameter, the risk of symptomatic myelopathy increases. ${ }^{63}$

\section{Magnetic Resonance Imaging}

Early OPLL appears slightly hyperintense on MR imaging without contrast administration, and inhomogeneously enhances with Gd-DTPA administration compared with disc herniations, which are uniformly hypointense. Between 30 and $60 \%$ of OPLL patients have accompanying disc protrusions. ${ }^{20,36,56}$ Early OPLL originates opposite multiple interspaces, which may be visualized on transaxial, coronal, and sagittal MR studies. These examinations provide an overview extending from the cervicomedullary through the cervicothoracic junction. Mature OPLL appears densely hypointense on both $\mathrm{T}_{1}$ - and $\mathrm{T}_{2}$-weighted MR studies, which demonstrate hyperintense foci within the ossified ligament indicating foci of active bone marrow production in mature haversian canals in $50 \%$ of patients with continuous OPLL. ${ }^{56}$ Greater detail of intrinsic changes in the cord may be seen on $\mathrm{T}_{2}$-weighted MR studies; hyperintense foci reflecting edema, myelomalacia, or gliosis may also be seen. These factors constitute poor prognostic indicators for patients with spondylotic myelopathy compared with OPLL-related myelopathy. ${ }^{3,56}$ Enhanced MR studies may differentiate postoperative scarring from disc intrusions and may also confirm the adequacy of postoperative decompression (Fig. 1). Accompanying vascular anomalies are readily revealed by MR angiography.

\section{Computerized Tomography Evaluations}

Early OPLL may contain punctate ossification or pearls of ossification on CT studies, and these centers may progressively coalesce as maturation occurs (Figs. 2 and 3) ${ }^{64}$ The four types of OPLL include: the segmental form (39\%) found behind the VBs; the continuous form (27\%) extending from vertebra to vertebra; the mixed form (29\%) including both continuous and segmental elements; and the other form (5\%) located opposite the disc spaces with some rostral and caudal retrovertebral extension (Figs. 4 and 5). Reconstructed 2D- and 3D-CT, and myelo-CT scans provide greater detail of compressive changes in the spinal cord attributable to ventral OPLL, whereas dynamic studies often reveal ventral intrusion from kyphosis or dorsal compromise resulting from ossification of the yellow ligament, facet arthropathy, or laminar shingling (Fig. 6).,11-14,20,22,25,30,31 Postoperative myelo-CT studies can be obtained to further document whether the cord has been adequately decompressed. ${ }^{43}$

\section{Documentation of Fusion by CT Scanning}

Fusion is typically documented on x-ray studies where trabeculation and the lack of osseous lucency may be con-

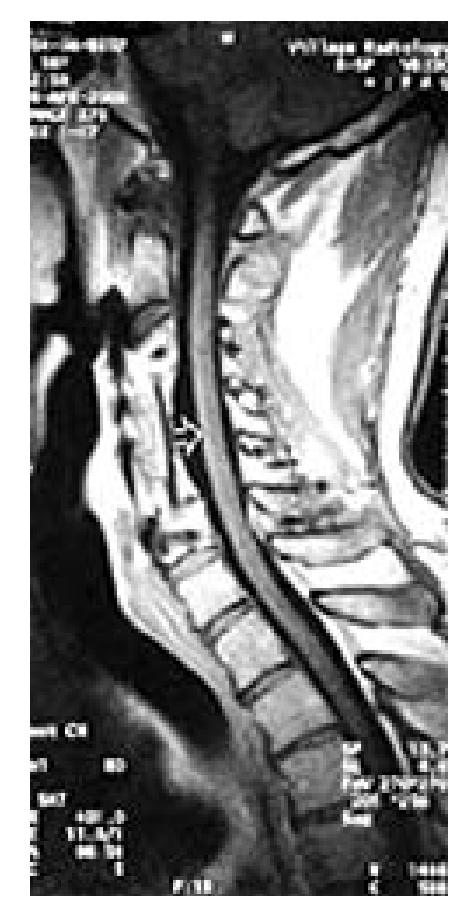

Fig. 1. Postoperative Gd-DTPA-enhanced $\mathrm{T}_{1}$-weighted MR image demonstrating adequate ventral cord decompression (arrow) 4 years after this patient underwent a $\mathrm{C} 3-6 \mathrm{ACF}$ and $\mathrm{C} 2-7 \mathrm{PWF}$ with an iliac crest autograft and an Orion plate. 


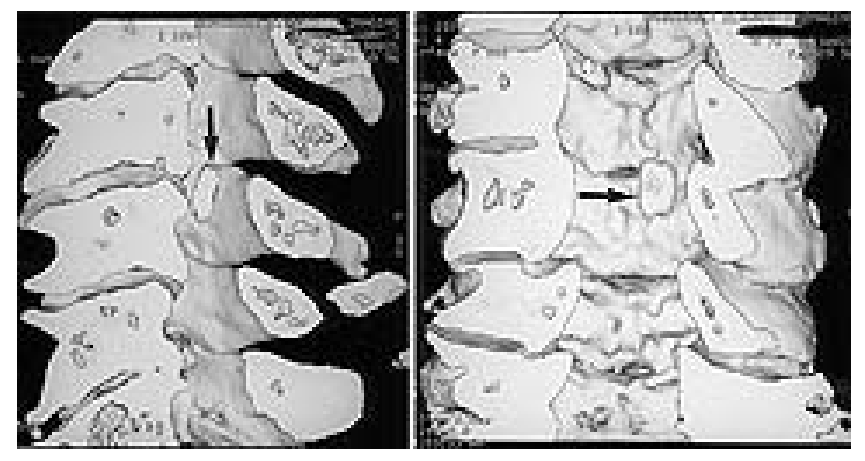

Fig. 2. Left: preoperative midline sagittal 3D-CT scan obtained in a 70-year-old man demonstrating a pearl of ossification (arrow) that was contained within a hypertrophied PLL opposite the C3-4 disc space. Note how this markedly affected the anteroposterior diameter of the cervical canal. Right: a coronal 3D-CT scan obtained in the same patient, demonstrating the pearl of ossification (arrow) at the C3-4 level that contributed to right-sided cord compression.

firmed at the graft-VB interface. Dynamic radiographs confirm the absence of motion, ideally less than $1 \mathrm{~mm}$ between the tips of contiguous spinous processes, and the lack of subluxation or translation. Also, 2D-CT studies may provide a third criterion for fusion following allograft fibula strut placement by documenting cephalad and caudad bone ingrowth into the central fibular canal (Fig. 7). ${ }^{23}$ In a recent personal series, ${ }^{23} 18$ patients undergoing $\mathrm{ACF} /$ PWF at an average of three levels, bone ingrowth was shown in 17 (94\%) of the 18 from 3 to 6 months postoperatively at a rate of $1.5 \mathrm{~mm}$ to $3.5 \mathrm{~mm}$ cephalad and $2.1 \mathrm{~mm}$ to $4.6 \mathrm{~mm}$ caudad.

\section{Computerized Tomography Signs of Dural Penetrance}

Ossification of the PLL extending through the dura is rarely seen in the North American population; it was found in only three of our 65 OPLL cases. ${ }^{15,27,36,39}$ In these patients, CT examinations offered two signs of potential dural penetrance. The single-layer sign on CT scans was characterized by a solid ossified ventral OPLL mass, often accompanied by a lateral "C-sign," indicating imbrication

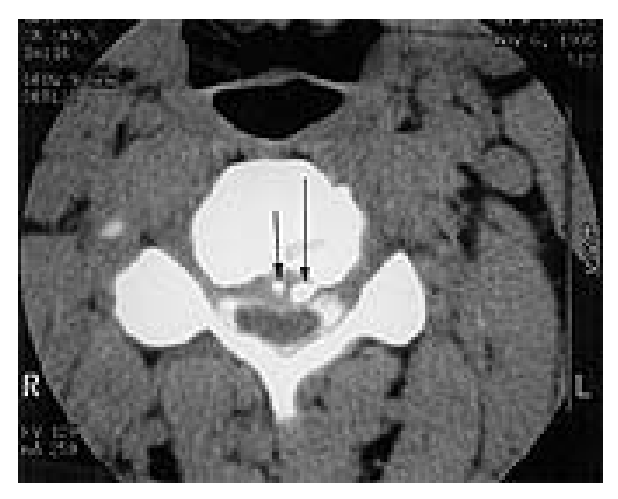

Fig. 3. A transaxial myelo-CT study obtained at the C4-5 level, demonstrating early OPLL, punctuate ossification (short arrow), and a pearl of ossification (long arrows).

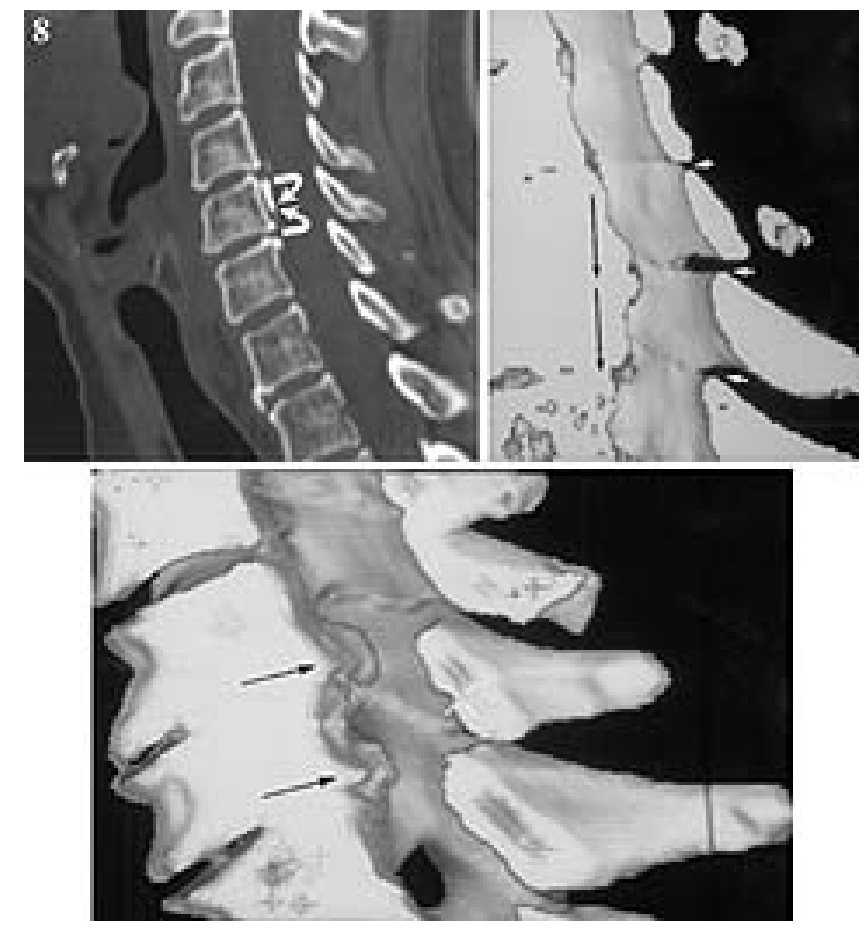

Fig. 4. Upper left: midline sagittal 2D-CT study revealing, segmental OPLL directly behind the VB (double arrows). Upper right: paramedian sagittal 3D-CT scan demonstrating continuous OPLL extending across the dorsal C-4 and C-5 VB (black arrows) accompanied by kyphosis seen as the exaggerated separation of the laminae (white arrows). Lower: paramedian 3D-CT study revealing OPLL involving the C5-6 and C6-7 levels (arrows) with retrovertebral extension crossing the dorsal aspect of the C-6 VB.

of the dura (Fig. 8). ${ }^{27}$ In cases of the single-layer sign, ossification appears to occur from the inside-out of the PLL. Hida, et al. ${ }^{46}$ observed an 11 to $25 \%$ correlation of absent dura at surgery when OPLL occupied more than $50 \%$ of the spinal canal. Only one of their nine patients in whom this single-layer sign was demonstrated developed a CSF leak during surgery. ${ }^{46}$

The double-layer sign, typified by a reversal of the ossification process from the outside-in, appears as a rim of OPLL surrounding the hypodense dura (Fig. 9). ${ }^{27}$ The double-layer sign has proven the most pathognomonic for absent dura at surgery; it correlated with absent dura in 10 of 12 patients reported on by Hida, et al. ${ }^{46}$

\section{Diffuse Idiopathic Skeletal Hyperostosis}

The syndrome know as DISH, which is characterized by ossification along the ventral aspect of the cervical vertebrae, is found in 15 to $30 \%$ of adults older than 65 years of age ${ }^{53,69}$ OPLL accompanies DISH up to $50 \%$ of the time ${ }^{33,61}$ Although DISH often becomes extensive, it rarely produces dysphagia, and careful diagnostic consideration should be given to other causes before considering resection with its attendant morbidity. ${ }^{61,67}$

\section{Ossification of the Anterior Longitudinal Ligament}

It is thought that OALL may contribute to dysphagia. The ossified mass itself appears hypointense on $\mathrm{T}_{1}$ - 


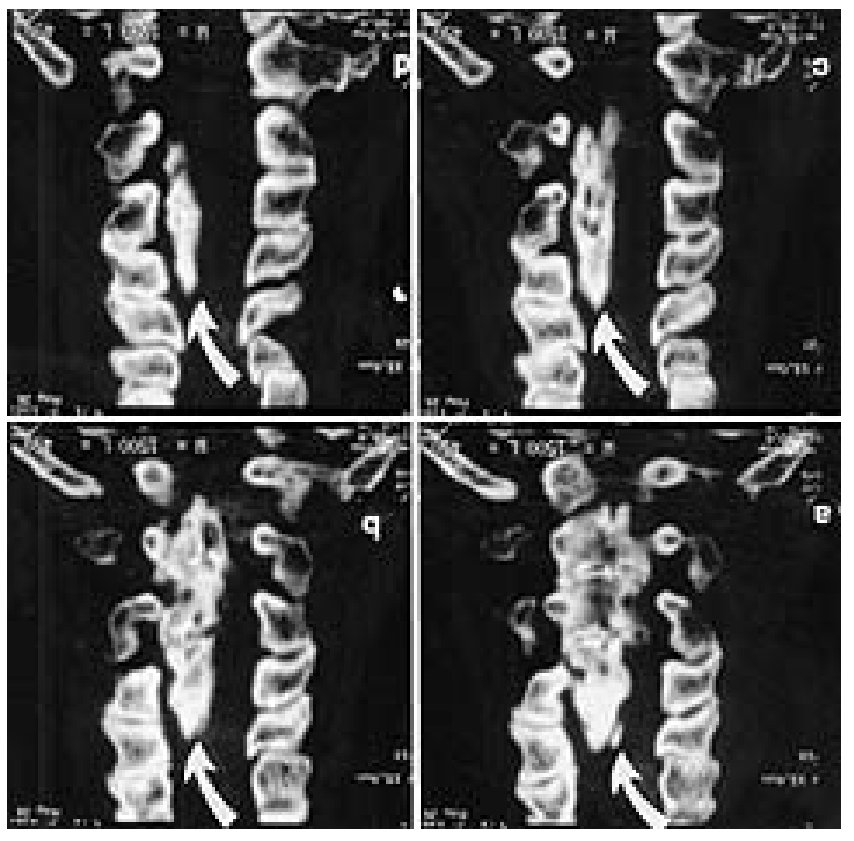

Fig. 5. Coronal 2D-CT study obtained at the $\mathrm{C} 2-7$ levels in four successive slices [(a) $2 \mathrm{~mm}$; (b) $4 \mathrm{~mm}$; (c) $6 \mathrm{~mm}$; and (d) $8 \mathrm{~mm}$ into the spinal canal] beginning at the ventral aspect of the cervical spinal canal extending dorsally revealing massive continuous OPLL.

and $\mathrm{T}_{2}$-weighted MR studies, whereas areas of bone marrow production appear hyperintense on $\mathrm{T}_{1}$-weighted images. ${ }^{40}$ At surgery, the adequacy of OALL resection may be checked on intraoperative lateral radiographs or fluoroscopy.

\section{CONSERVATIVE COMPARED WITH SURGICAL MANAGEMENT OF OPLL PATIENTS}

Most older patients ( $>65$ years of age) with asymptomatic OPLL but with significant medical comorbidities should be followed conservatively because prophylactic surgery in this age group plays a minimal role. For those with rapidly progressive myelopathy and several comorbidity factors, including cardiovascular disease, chronic obstructive pulmonary disease, diabetes, and peripheral vascular disease, nonsurgical management should be strongly considered because the risks of perioperative morbidity and mortality increase. In the series by Saunders, et al. ${ }^{71}$ three of 31 postoperative deaths occurred in patients older than 70 years of age with significant cardiovascular comorbidities. Similarly, in my series ${ }^{20,36}$ of 44 patients undergoing multilevel ACF/PWF, two deaths occurred in patients with significant cardiac disease. ${ }^{20,36}$ Patients with severe, long-standing myelopathy (Nurick Grade $\mathrm{V}$ ) and increased cord signals on $\mathrm{T}_{2}$-weighted MR images, reflecting myelomalacia (apoptosis) and cord atrophy, are also poor candidates for surgery.

Alternatively, those patients younger than 65 years of age, without fixed deficits or major comorbidities, should be considered candidates for prophylactic surgery in the face of CT- or MR-documented severe compromise of the spinal cord. Surgery performed in these cases, prior to

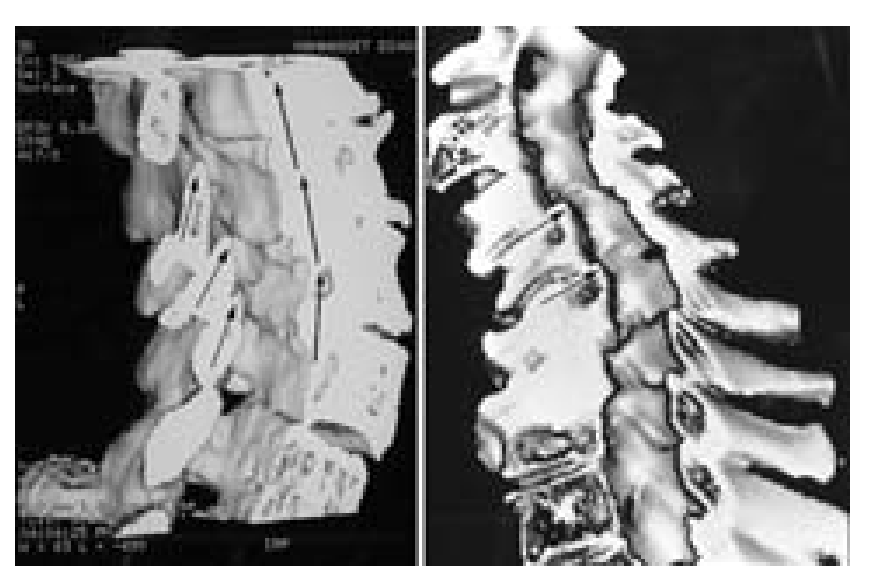

Fig. 6. Left: midline sagittal 3D-CT study revealing C2-C5 OPLL (longer arrows) ventrally and marked shingling of the C35 laminae (shorter arrows) dorsally. Right: paramedian sagittal 3D-CT scan demonstrating ventral kyphosis attributed to marked degenerative subluxation of the C4-5 and C5-6 VB (arrows).

even minor cervical trauma, may avoid future quadriparesis or quadriplegia. ${ }^{42,50}$

\section{ANTERIOR SURGERY FOR OPLL}

Direct anterior resection of OPLL results in improved postoperative outcomes. In the study by Fessler, et al., ${ }^{41}$ Nurick grades improved $86 \%$ of the time following anterior surgical approaches. These patients improved an average of 1.24 Nurick grades, whereas those undergoing laminectomy improved only 0.07 Nurick grades.

Better clinical outcomes are encountered following anterior resection rather than posterior decompression of OPLL. 12,14,20,35,36 The patients reported on by Kawano, et al. ${ }^{51}$ showed an overall mean improvement rate on the Neurosurgical Cervical Spine Scale score of $78 \%$ for anterior compared with $46.1 \%$ for posterior decompressions.

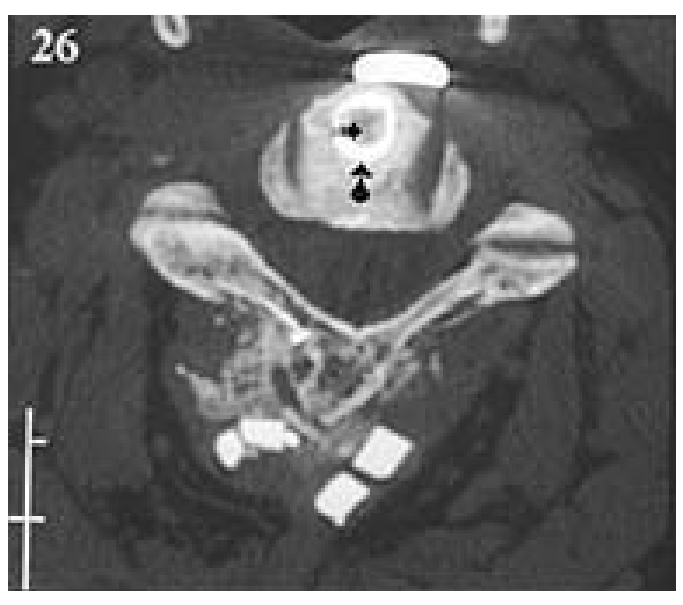

Fig. 7. Six-month postoperative transaxial noncontrast-enhanced CT scan demonstrating osseous ingrowth into the central fibular canal (interrupted arrow) accompanied by bone trabeculation and the lack of osseous lucency at the external fibula-VB junction (rounded arrow). 


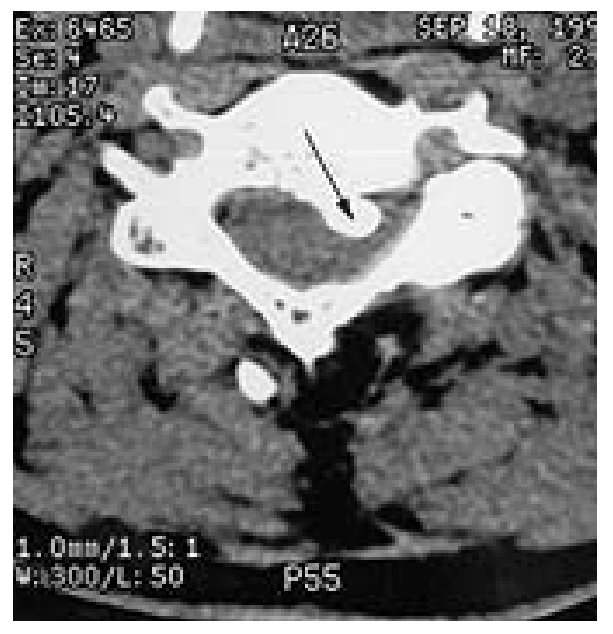

Fig. 8. Transaxial noncontrast-enhanced CT study revealing, the combined "single layer" and "C" signs (arrow) that contributed to left-sided cord compression and imbrication of the dura, which indicates greater potential for an intraoperative CSF fistula.

Long-term outcomes were also superior following anterior resection of OPLL; scores for those undergoing anterior resection continued to rise postoperatively to 13 whereas for those undergoing laminoplasty a progressive decline to 9.7 was seen.

\section{One-Level ACF}

When OPLL involves two contiguous interspaces with significant retrovertebral extension, it is best resected with a one level ACF, with excision of portions of the cephalad and caudad VBs, two discs, and the intervening vertebra. One-level ACF requires fusion at two graft-VB interfaces rather than the four required for two-level $\mathrm{ADF}^{7,16}$ Epstein ${ }^{16}$ reported fusion rates of $99 \%$ for 78 patients undergoing nonplated one-level ADF, but only a $90 \%$ success rate for those undergoing nonplated two-level ADF. Emery, et al., ${ }^{9}$ observed a $16 \%$ pseudarthrosis rate following one- to two-level nonplated ADF. Plating reduced graft extrusion, kyphosis, and pseudarthrosis rates following one- and 2-level ADF, with some citing $100 \%$ fusion rates. ${ }^{5,7,15,29}$

\section{Dynamic Plating of One-Level ACF: Personal Series}

A greater success rate was found following dynamic rather than fixed-plate in one-level ACF performed for OPLL. Operative failures, defined as graft-plate extrusion or pseudarthrosis, were studied following 57 one-level ACF surgeries. Fixed plates (Orion Plates and Atlantis Plates; Sofamor Danek, Memphis, TN) placed in the first 15 patients resulted in seven failures: one plate extrusion, three pseudarthroses, and three delayed midstrut fractures. Dynamic plates (ABC; Aesculap, Tuttlingen, Germany) applied in 42 patients resulted in four failures: one plategraft extrusion, one pseudarthrosis, and two delayed midstrut fractures. ${ }^{24}$ The placement of dynamic ABC plates allowed for up to $10 \mathrm{~mm}$ of cephalad and caudad migration of the plates over the screw heads within longitudinal grooves in the plates. Using dynamic plates, cephalad mi-

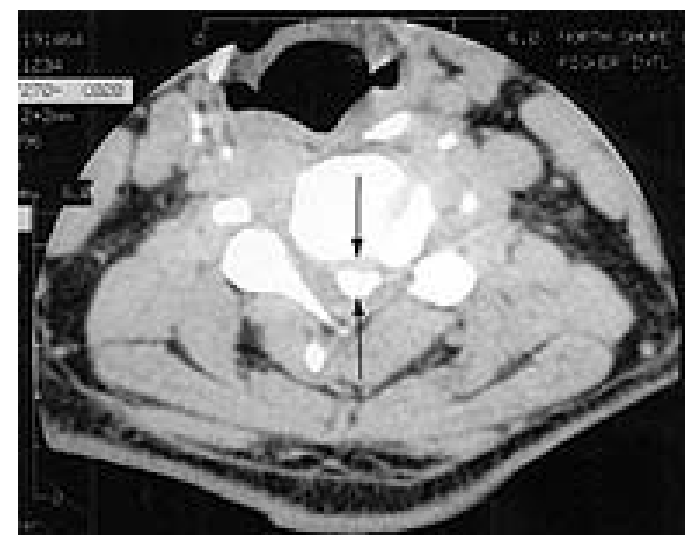

Fig. 9. Noncontrast-enhanced transaxial CT scan obtained at the C5-6 level, revealing dural penetration characterized by the "double-layer" sign; a large mass of ossification is seen within the spinal canal separated from the dorsal aspect of the VB by residual hypodense dura (arrows).

gration averaged $6.7 \mathrm{~mm}$ (range $3-10 \mathrm{~mm}$ ), and caudad migration averaged $5.8 \mathrm{~mm}$ (range $3-8 \mathrm{~mm}$ ). The heightened success when using the dynamic plates was variously attributed to their reduction of stress shielding and the allowance for greater compression, which facilitated stable fusion.

\section{Multilevel ACF}

More typically, OPLL involves several segments, warranting multilevel ACF. Ventral approaches to OPLL resulting in direct resection are correlated with better long-term outcomes, although immediate perioperative morbidity may prove higher. ${ }^{12,13,15,19-22,36,39,52,62,75}$ Epstein $^{25}$ noted that in 76 OPLL patients undergoing nonplated $\mathrm{ADF}$ (mean 3.5 levels) or ACF (mean 3 levels) that $13 \%$ developed pseudarthrosis/instability within 6 months of surgery. In $10 \%$ of the patients reported by Saunders, et al., ${ }^{71}$ nonplated four-level ACF failed acutely. In another series of patients undergoing two- to four-level ACF performed with and without plates, a combined perioperative mortality and major morbidity rate of $22 \%$ was noted, despite an ultimate $97 \%$ fusion rate. ${ }^{58}$ A $9 \%$ failure rate was observed by Vaccaro, et al. ${ }^{75}$ when using fixed plates to perform two-level ACF, the rate increased to $50 \%$ with three-level ACF. Eleraky, et al., ${ }^{8}$ surprisingly found a $3.2 \%$ neurological complication rate, $98.8 \%$ fusion rate, and $86.5 \%$ improvement rate after having used plates in one-level ACF (87 patients) and two- to three-level ACF (98 patients).

\section{Results Using Fixed Compared With Dynamic Plates}

Lower complication rates were associated with using dynamic $\mathrm{ABC}$ plates for multilevel constructs combined with PWF. Two of 22 multilevel (two-four levels) ACF/ PWF procedures performed without the benefit of anterior plates resulted in three immediate postoperative graft extrusions ${ }^{20}$ Fixed Orion plates used in the next 22 multilevel circumferential constructs resulted in two immediate inferior graft-plate extrusions. ${ }^{21,36}$ Applying Atlantis plates in 16 multilevel $\mathrm{ACF} / \mathrm{PWF}$ procedures resulted in 


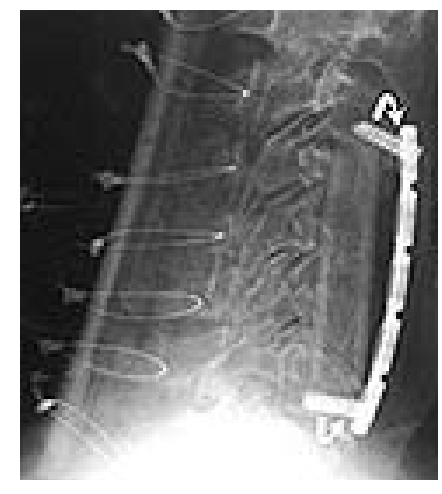

Fig. 10. Lateral cervical x-ray film obtained 6 months following a C3-7 ACF and C2-T1 PWF with dynamic ABC plate placement; 5-mm cephalad and 4-mm caudad plate migration was observed (arrows).

three extrusions observed between 1 month (two patients), and 4 months postoperatively; all were inferior graft, plate, and screw extrusions. ${ }^{27}$ Twenty-six multilevel ACF/ PWF procedures performed with dynamic plates resulted in only one patient manifesting a "partial" pseudarthrosis of the anterior graft viewed on sequential CT studies; this patient required secondary PWF (Fig. 10). ${ }^{24}$ The average dynamic plate migration after multilevel $\mathrm{ACF} / \mathrm{PWF}$ was $6.3 \mathrm{~mm}$ (range $4-10 \mathrm{~mm}$ ) cephalad and $5.9 \mathrm{~mm}$ (range 4-9 $\mathrm{mm}$ ) caudad. Dynamic plating similarly appeared to limit stress shielding, promoted graft settling, and fostered fusion in multilevel constructs. ${ }^{26}$

\section{Posterior Fusion Techniques}

Posterior fusions may include wiring of spinous processes or facets, or the use of lateral mass or pedicle screws with plates or rods. The addition of the dorsal fusion to provide a posterior "tension band" following multilevel ACF has been well-documented by Kirkpatrick, et al. ${ }^{52}$ They performed a biomechanical study in the sagittal plane and found that posterior fusion reduced the range of motion by $62 \%$, compared with $24 \%$ with strut grafting only and $43 \%$ if an anterior strut graft and plate were applied.

\section{Anterior Floating Method: an Option for Anterior OPLL Resection}

The anterior floating method as an alternative to direct ventral OPLL resection may be offered, particularly when the OPLL fills more than $60 \%$ of the canal. ${ }^{77}$ It requires more extensive lateral exposure $(<25 \mathrm{~mm})$ to facilitate anterior migration of the OPLL mass and, therefore, poses a significant increased risk of vertebral artery and nerve root injuries. The lack of direct OPLL resection may likely lead to continued cord compression and pose the risk of ventral retethering.

\section{COMPLICATIONS OF MULTILEVEL ACF/PWF}

\section{Vertebral Artery Injury}

Endovascular repair of vertebral artery injuries sustained in the course of cervical surgery is preferred. For those injuries less than 6 hours old, stent placement is feasible, whereas for injuries more than 6 hours old, trapping should be performed to occlude the vessel and avoid cephalad progression of a thrombus. Older techniques, including direct surgical repair of the vessel or indirect pressure/occlusion risk greater morbidity to the patient.

\section{Cord and Root Injuries}

A 2 to $10 \%$ incidence of quadriplegia and an approximately 5 to $17 \%$ incidence of root injury (typically C-5) has been reported in cervical surgery. ${ }^{81}$ Root injuries are less frequently attributed to direct manipulation than to rapid cord migration or the so-called untethering effect. ${ }^{71}$ To limit these complications, Saunders, et al. ${ }^{71}$ recommended limiting the anterior trough to $15 \mathrm{~mm}$ and found that the frequency of root injuries was markedly reduced. The interpedicular distance, however, in many North American patients measures from 18 to $20 \mathrm{~mm}$ and a 15$\mathrm{mm}$ decompression will prove inadequate.

\section{POSTERIOR SURGERY}

High-risk patients older than 65 years of age with OPLL but with adequate cervical lordosis may undergo posterior decompressive procedures, including laminectomy, laminectomy with posterior fusion, or laminoplasty, with a modicum of success (Fig. 11). 4,18,28,45,48,49,57,76,80 The lordosis allows the cord to migrate away from ventrally situated disease, whereas kyphosis can result in tethering of the cord over ventral OPLL protrusions.

\section{Laminectomy}

Sufficient cord decompression may be accomplished by laminectomy alone when there is no evidence of spinal instability. If medial facetectomies and foraminotomies

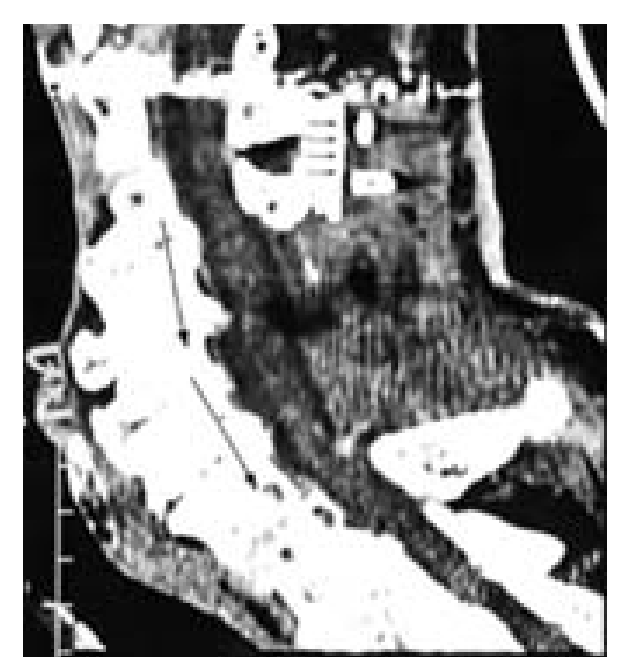

Fig. 11. A CT study obtained in a 78-year-old patient in whom a cervical laminectomy was performed from C-4 to C-7 for multilevel OPLL, spondylosis, and stenosis (long arrows), along with a C2-C3 PWF (short arrows) for focal instability. Note how multilevel OALL (curved arrows) contributed to spontaneous ventral fusion from C-3 to C-6 (long arrows), and OPLL resulted in fusion from $\mathrm{C}-3$ to $\mathrm{T}-1$. 
are restricted to less than $25 \%$ of the facet joint, stability may be preserved, whereas facet removal of $50 \%$ or greater will likely result in destabilization. ${ }^{66}$ Long-term neurological recovery rates of $44.2 \%$ at 1 year and $42.9 \%$ at 5 years following laminectomy have been documented, although outcomes typically deteriorate between 5 and 10 years postoperatively. ${ }^{49}$ Poor prognostic factors include advanced deficits, trauma, and ossification of the yellow ligament, but rarely OPLL progression.

\section{Laminectomy With Posterior Fusion}

Laminectomy and posterior fusion may also be used to manage cervical OPLL in the presence of adequate lordosis. Postlaminectomy instability may also be avoided with the addition of posterior stabilization (Fig. 12). ${ }^{66}$

\section{Posterior Fusion Techniques}

Posterior fusion alternatives include facet and/or spinous process wiring techniques, lateral mass plates, and pedicle screws, the latter using rod/plate systems., ${ }^{1,28,28,}$ 45,57,76 Epstein ${ }^{28,32}$ performed spinous process wiring and fusion successfully in 65 patients undergoing multilevel cervical ACF/PWF for stabilization; more recently this included 86 patients. Comparable neurological outcomes were observed in patients undergoing laminectomy (without instability) and laminectomy with fusion (for instability) in patients with cervical OPLL. ${ }^{45}$ Using the Short Form 36, patient-based outcome data for 25 patients revealed adequate postoperative results following laminectomy with lateral mass plating for unstable spondylotic myelopathy. ${ }^{57}$ No symptomatic postoperative instability or

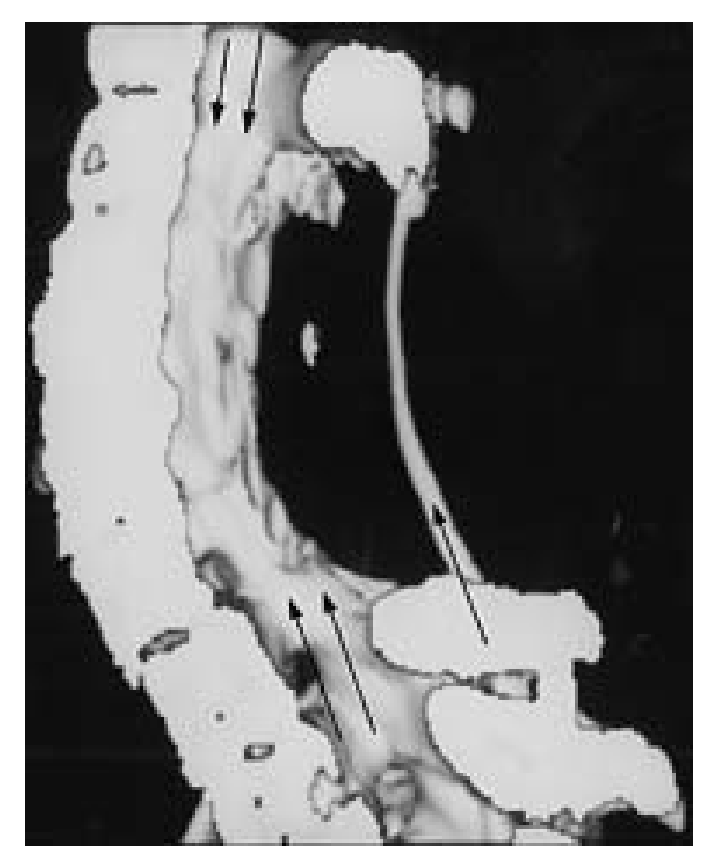

Fig. 12. A 3D-CT study revealing cervical stenosis accompanied by mild ventral OPLL and an adequately preserved cervical lordotic curvature that were treated with a C3-7 laminectomy (double arrows) and $\mathrm{C} 2-\mathrm{T} 1$ facet wiring and fusion by using braided cables placed from C-2 to T-1 (single arrow). increases in kyphosis were observed: $80 \%$ had good outcomes and none deteriorated. Lateral mass plates were similarly successful in the management of postlaminectomy instability; no significant complications were noted in the placement of 281 screws. $^{76}$ Dorsal decompression with or without fusion may not suffice in some patients with significant anterior disease and secondary anterior procedures are necessary. ${ }^{1}$ Nevertheless, the use of lateral mass screws may result in "near misses" as shown in the postoperative study by Abumi, et al., ${ }^{2}$ who reported an incidence of screws perforating the cortex of the pedicles in 10 of 190 cases $(5.3 \%)$. Long-term recovery rates of $44.2 \%$ were found after 1 year and were even maintained at $42.9 \%$ after 5 years; deterioration appeared between 5 and 10 years postoperatively at a rate of $32.8 \% .^{49}$

\section{ANESTHETIC PROTOCOL FOR CIRCUMFERENTIAL CERVICAL SURGERY}

Circumferential cervical procedures with halo devices were performed to address multilevel OPLL. Patients were managed with a strict anesthetic protocol to avoid emergency reintubation or tracheostomy, which could result in acute graft and plate disruption, increased neurological deficit, hypoxia, and death. ${ }^{10,17}$ Awake fiberoptic intubation and positioning using continuous intraoperative SSEP monitoring is performed. Patients continue on intubation during the 1st postoperative night, and are extubated with fiberoptic assistance thereafter by a qualified anesthesiologist. ${ }^{17}$ Prior to extubation a patient must fullfil the following criteria: 1) direct fiberoptic evaluation of the trachea and vocal cords for swelling; 2) indirect assessment of swelling performed by letting down their endotracheal cuff checking for an air leak and the ability to verbalize; and 3) thorough review of the patients' attendant medical risk factors. Patients who are unable to undergo extubation by the 7 th postoperative day receive prophylactic tracheostomies performed by ear, nose, and throat specialists. Fifty-eight patients who underwent an average three-level ACF and 6.5-level PWF for OPLL were managed using this strict protocol. ${ }^{17}$ Operative times were approximately 10 hours and required 600 to $800 \mathrm{ml}$ of blood transfusion. Forty patients underwent fiberoptically assisted extubation on the 1st postoperative day, 15 on Days 2 through 7, and three required elective tracheostomy on the 7 th day. Following this protocol, only one patient underwent emergency reintubation 20 minutes after having undergone extubation on postoperative Day 3. She had undergone a C4-7 ACF 3 years previously, was asthmatic, and her surgery spanned 14 hours, requiring both wound-peritoneal and lumboperitoneal shunt placement for a CSF fistula.

Seven major risk factors and complications correlated with delays in extubation or tracheostomy. These included in descending order: 1) operative time more than 10 hours (12 patients); 2) obesity greater than 220 lbs (12 patients); 3) transfusions of more than 1000 to $1200 \mathrm{ml}$ of blood (10 patients); 4) secondary anterior cervical surgery (nine patients); 5) anterior surgery including the $\mathrm{C}$-2 level (seven patients); 6) four-level ACF (five patients); and 7) asthma (five patients). Minor risk factors included: 1) advanced age ( $>65$ years old $) ; 2$ ) severe preoperative neu- 
rological deficits (Nurick Grade IV-V moderate/severe myelopathy); and 3) intraoperative CSF fistula. Factors cited in the literature as contributing to airway complications included angioedema, recurrent laryngeal nerve palsy, dysphagia with or without esophageal perforation, and new spinal cord injuries. ${ }^{9,81}$

\section{MONITORING OF SSEPS AND MEPS}

Intubation and positioning while the patient is awake, and continuous intraoperative SSEP monitoring of median/ulnar and posterior tibial potentials during cervical procedures, including OPLL surgery, limits postoperative morbidity. ${ }^{34,37,60}$ A $50 \%$ decrease in amplitude and a $10 \%$ decline in latency are significant. ${ }^{37}$ Changes observed at 50 seconds may be reproduced within 100 seconds and require immediate resuscitative measures. Medical measures include the inducing of hypertension, warming of irrigating fluids, decreasing the concentration of inhalation anesthetic, and increasing oxygen concentration. Surgical resuscitative techniques include releasing distraction, removal of an oversized graft, and cessation of manipulation. A deterioration in SSEPs may signal flexion or extension of the neck, traction, or rotation or compression at the level of the shoulder, elbow or wrist. To avoid SSEP "drop out," inhalation anesthetics (for example, isoflorane) are usually maintained at concentrations below $0.4 \%$ or a balanced narcotic technique is used. In a prospective study, Epstein and colleagues ${ }^{37}$ found no cases of quadriplegia in 100 SSEP-monitored cases compared with eight of 218 unmonitored patients who became quadriplegic during the course of surgery performed by five different neurosurgeons. May and coworkers ${ }^{60}$ monitored SSEPs during 182 cervical procedures; however, 10 patients developed new postoperative deficits. Major risk factors associated with greater deficits included: 1) multisegmental surgery; 2) more severe preoperative neurological status; and 3) placement of instrumentation. Half of the patients in whom complete SSEP signals were lost during surgery developed new postoperative deficits, whereas those in whom incomplete drop out of SSEP signal occurred or those with intraoperative recovery of SSEPs did not. False positives are more likely to be true "mini" positives, permanent deficits being avoided by the immediate institution of appropriate resuscitative measures. Monitoring of MEPs also provide helpful information regarding the status of the anterior spinal cord. Gokaslan and colleagues ${ }^{44}$ transcutaneously placed electrodes for MEP monitoring during 16 anterior cervical fusion operations and with no complications were able to preserve MEPs, which correlated with good clinical outcomes.

\section{References}

1. Abumi K, Kaneda K: Pedicle screw fixation for nontraumatic lesions of the cervical spine. Spine 22:1853-1863, 1997

2. Abumi K, Kaneda K, Shono Y, et al: One-stage posterior decompression and reconstruction of the cervical spine by using pedicle screw fixation systems. J Neurosurg 90 (Spine 1): 19-26, 1999

3. Baba H, Furusawa N, Imura S, et al: Late radiographic findings after anterior cervical fusion for spondylotic myeloradiculopathy. Spine 18:2167-2173, 1993
4. Baisden J, Voo LM, Cusick JF, et al: Evaluation of cervical laminectomy and laminoplasty. A longitudinal study in the goat model. Spine 24:1283-1289, 1999

5. Bose B: Anterior cervical fusion using Caspar plating: analysis of results and review of the literature. Surg Neurol 49:25-31, 1998

6. Breitbart AS, Grande DA, Kessler R, et al: Tissue engineered bone repair of calvarial defects using cultured periosteal cells. Plastic Reconstr Surg 101:567-576, 1998

7. Connolly PJ, Esses SI, Kostuik JP: Anterior cervical fusion: outcome analysis of patients fused with and without anterior cervical plates. J Spinal Disord 9:202-206, 1996

8. Eleraky MA, Llanos C, Sonntag VK: Cervical corpectomy: report of 185 cases and a review of the literature. J Neurosurg 90 (Spine 1):35-41, 1999

9. Emery SE, Bohlman HH, Bolesta MJ, et al: Anterior cervical decompression and arthrodesis for the treatment of cervical spondylotic myelopathy. Two to seventeen-year follow-up. J Bone Joint Surg Am 80:941-951, 1998

10. Emery SE, Smith MD, Bohlman HH: Upper-airway obstruction after multilevel cervical corpectomy for myelopathy. J Bone Joint Surg Am 73:544-551, 1991

11. Epstein N: Diagnosis and surgical management of ossification of the posterior longitudinal ligament. Contemp Neurosurg 14:1-6, 1992

12. Epstein N: The surgical management of ossification of the posterior longitudinal ligament in 51 patients. J Spinal Disord 6: 432-454, 1993

13. Epstein N: Vertebral body fractures following extensive anterior cervical surgical procedures for ossification of the posterior longitudinal ligament. Neuroorthopedics 21:1-11, 1997

14. Epstein NE: Advanced cervical spondylosis with ossification into the posterior longitudinal ligament and resultant neurologic sequelae. J Spinal Disord 9:477-484, 1996

15. Epstein NE: Anterior approaches to cervical spondylosis and ossification of the posterior longitudinal ligament: review of operative technique and assessment of 65 multilevel circumferential procedures. Surg Neurol 55:313-324, 2001

16. Epstein NE: Anterior cervical diskectomy and fusion without plate instrumentation in 178 patients. J Spinal Disord 13:1-8, 2000

17. Epstein NE: Can airway complications following multilevel anterior cervical surgery be avoided? J Neurosurg 94 (Spine 2): 185-188, 2001

18. Epstein NE: Cervical posterior approaches in the management of spondylosis and ossification of the posterior longitudinal ligament. Surg Neurol (In press)

19. Epstein NE: Circumferential cervical surgery for spondylostenosis with kyphosis in two patients with athetoid cerebral palsy. Surg Neurol 52:339-344, 1999

20. Epstein NE: Circumferential surgery for the management of cervical ossification of the posterior longitudinal ligament. J Spinal Disord 11:200-207, 1998

21. Epstein NE: A comparative analysis of plate/graft failure with correction following circumferential cervical spinal surgery. Spinal Surg 16:1-8, 2002

22. Epstein NE: Complications following surgical procedures of ossification of the posterior longitudinal ligament in the cervical spine warranting additional surgical correction. Neuroorthopedics 22:85-97, 1998

23. Epstein NE: Computed Tomography (CT) validating bony ingrowth into fibula strut allograft: A criterion for fusion. Spine J (In press)

24. Epstein NE: The efficacy of anterior dynamic plates in complex cervical surgery. J Spinal Disord (In press)

25. Epstein NE: Evaluation and treatment of clinical instability associated with pseudarthrosis after anterior cervical surgery for ossification of the posterior longitudinal ligament. Surg Neurol 49:246-252, 1998 
26. Epstein NE: From postsurgical imaging: marked superior and inferior migration of a dynamic plate after multilevel anterior corpectomy and fusion with posterior wiring. Spine J 1:226, 2001

27. Epstein NE: Identification of ossification of the posterior longitudinal ligament extending through the dura on preoperative computed tomographic examination of the cervical spine. Spine 26:182-186, 2001

28. Epstein NE: Laminectomy with posterior wiring and fusion for cervical ossification of the posterior longitudinal ligament, spondylosis, ossification of the yellow ligament, stenosis, and instability: a study of 5 patients. J Spinal Disord 12:461-466, 1999

29. Epstein NE: The management of one-level anterior cervical corpectomy with fusion using Atlantis hybrid plates: preliminary experience. J Spinal Disord 13:324-328, 2000

30. Epstein NE: Ossification of the posterior longitudinal ligament: diagnosis and surgical management. Neurosurg Quart 2: 223-241, 1992

31. Epstein NE: Ossification of the posterior longitudinal ligament in evolution in 12 patients. Spine 19:673-681, 1994

32. Epstein NE: Reoperation rates for acute graft extrusion and pseudarthrosis after one level anterior corpectomy and fusion with and without plate instrumentation: etiology and corrective management. Surg Neurol 56:73-81, 2001

33. Epstein NE: Simultaneous cervical diffuse idiopathic skeletal hyperostosis and ossification of the posterior longitudinal ligament resulting in dysphagia or myelopathy in two geriatric North Americans. Surg Neurol 53:427-431, 2000

34. Epstein NE: Somatosensory evoked potential monitoring (SSEPs) in 173 cervical operations. Neuroorthopedics 20: 3-21, 1996

35. Epstein NE: The surgical management of ossification of the posterior longitudinal ligament in 43 North Americans. Spine 19:664-672, 1994

36. Epstein NE: The value of anterior cervical plating in preventing vertebral fracture and graft extrusion following multilevel anterior cervical corpectomy with posterior wiring and fusion: indications, results, and complications. J Spinal Disord 13:9-15, 2000

37. Epstein NE, Danto J, Nardi D: Evaluation of intraoperative somatosensory-evoked potential monitoring during 100 cervical operations. Spine 18:737-747, 1993

38. Epstein NE, Grande DA, Breitbart AS: In vitro characteristics of cultured posterior longitudinal ligament tissue. Spine 27: 56-58, 2002

39. Epstein NE, Hollingsworth R: Anterior cervical micro-dural repair of cerebrospinal fluid fistula after surgery for ossification of the posterior longitudinal ligament. Technical Note. Surg Neurol 52:511-514, 1999

40. Epstein NE, Hollingsworth R: Ossification of the anterior longitudinal ligament (OALL) contributing to dysphagia. Case report. J Neurosurg 90 (Spine 2):261-263, 1999

41. Fessler RG, Steck JC, Giovanini MA: Anterior cervical corpectomy for cervical spondylotic myelopathy. Neurosurgery 43: 257-265, 1998

42. Fujimura Y, Nakamura M, Toyama Y: Influence of minor trauma on surgical results in patients with cervical OPLL. J Spinal Disord 11:16-20, 1998

43. Fujimura Y, Nishi Y, Nakamura M: Dorsal shift and expansion of the spinal cord after expansive open-door laminoplasty. J Spinal Disord 10:282-287, 1997

44. Gokaslan ZL, Samudrala, S, Deletis V, et al: Intraoperative monitoring of spinal cord function using motor evoked potentials via transcutaneous epidural electrode during anterior cervical spinal surgery. J Spinal Disord 10:299-303, 1997

45. Hamanishi C, Tanaka S: Bilateral multilevel laminectomy with or without posterolateral fusion for cervical spondylotic myelopathy: relationship to type of onset and time until operation. J Neurosurg 85:447-451, 1996
46. Hida K, Iwasaki Y, Kohanagi I, et al: Bone window computed tomography for detection of dural defect associated with cervical ossified posterior longitudinal ligament. Neurol Med Chir 37:173-176, 1997

47. Ikegawa S, Kurokawa T, Hizuka N, et al: Increase of serum growth hormone-binding protein in patients with ossification of the posterior longitudinal ligament of the spine. Spine 18: 1757-1760, 1993

48. Ishida Y, Ohmori K, Suzuki K, et al: Analysis of dural configuration for evaluation of posterior decompression in cervical myelopathy. Neurosurgery 44:91-96, 1999

49. Kato Y, Iwasaki M, Fuji T, et al: Long-term follow-up results of laminectomy for cervical myelopathy caused by ossification of the posterior longitudinal ligament. J Neurosurg 89: 217-223, 1998

50. Katoh S, Ikata T, Hirai N, et al: Influence of minor trauma to the neck on the neurological outcome in patients with ossification of the posterior longitudinal ligament (OPLL) of the cervical spine. Paraplegia 33:330-333, 1995

51. Kawano H, Handa Y, Ishii H, et al: Surgical treatment for ossification of the posterior longitudinal ligament of the cervical spine. J Spinal Disord 8:145-150, 1995

52. Kirkpatrick JS, Levy JA, Carillo J, et al: Recontruction after multilevel corpectomy in the cervical spine. A sagittal plane biomechanical study. Spine 24:1186-1191, 1999

53. Kissel P, Youmans JR: Posttraumatic anterior cervical osteophyte and dysphagia: surgical report and literature review. J Spinal Disord 5:104-107, 1992

54. Koga H, Hayashi K, Taketomi E, et al: Restriction fragment length polymorphism of genes of the $\alpha 2$ (XI) collagen, bone morphogenetic protein-2, alkaline phosphatase, and tumor necrosis factor- $\alpha$ among patients with ossification of posterior longitudinal ligament and controls from the Japanese population. Spine 21:469-473, 1996

55. Koga H, Sakou T, Taketomi E, et al: Genetic mapping of ossification of the posterior longitudinal ligament of the spine. Am J Hum Genet 62:1460-1467, 1998

56. Koyanagi I, Iwasaki Y, Hida K, et al: Magentic resonance imaging findings in ossification of the posterior longitudinal ligament of the cervical spine. J Neurosurg 88:247-254, 1998

57. Kumar VG, Rea GL, Mervis LJ, et al: Cervical spondylotic myelopathy: functional and radiographic long-term outcome after laminectomy and posterior fusion. Neurosurgery 44: 771-778, 1999

58. Macdonald RL, Fehlings MG, Tator CH, et al: Multilevel anterior cervical corpectomy and fibular allograft fusion for cervical myelopathy. J Neurosurg 86:990-997, 1997

59. Matsunaga S, Yamaguchi M, Hayashi K, et al: Genetic analysis of ossification of the posterior longitudinal ligament. Spine 24:937-939, 1999

60. May DM, Jones SJ, Crockard HA: Somatosensory evoked potential monitoring in cervical surgery: identification of pre- and intraoperative risk factors associated with neurological deterioration. J Neurosurg 85:566-573, 1996

61. McCafferty RR, Harrison MJ, Tamas LB, et al: Ossification of the anterior longitudinal ligament and Forestier's disease: an analysis of seven cases. J Neurosurg 83:13-17, 1995

62. Meding JB, Stambough JL: Critical analysis of strut grafts in anterior spinal fusions. J Spinal Disord 6:166-174, 1993

63. Miyasaka H: Consideration on pathophysiology of OPLL. Clin Orthop 10:1091-1096, 1975 (Reference unverified)

64. Morimoto T, Matsuyama T, Hirabayashi H, et al: Expansive laminoplasty for multilevel cervical OPLL. J Spinal Disord 10:296-298, 1997

65. Motegi H, Yamazaki M, Goto S, et al: Proliferating cell nuclear antigen in hypertrophied spinal ligaments. Immunohistochemical localization of proliferating cell nuclear antigen in hypertrophied posterior longitudinal ligament of the cervical spine. Spine 23:305-310, 1998 


\section{N. Epstein}

66. Nowinski GP, Visarius H, Nolte LP, et al: A biomechanical comparison of cervical laminaplasty and cervical laminectomy with progressive facetectomy. Spine 18:1995-2004, 1993

67. Oga M, Mashima T, Iwakuma T, et al: Dysphagia complications in ankylosing spinal hyperostosis and ossification of the posterior longitudinal ligament. Roentgenographic findings of the developmental process of cervical osteophytes causing dysphagia. Spine 18:391-394, 1993

68. Okada K, Shirasaki N, Hayashi H, et al: Treatment of cervical spondylotic myelopathy by enlargement of the spinal canal anteriorly, followed by arthrodesis. J Bone Joint Surg Am 73: 352-364, 1991

69. Resnick D, Guerra J Jr, Robinson CA, et al: Association of diffuse idiopathic skeletal hyperostosis (DISH) and calcification and ossification of the posterior longitudinal ligament. AJR 131:1049-1053, 1978

70. Sakou T, Taketomie E, Matsunaga S, et al: Genetic study of ossification of the posterior longitudinal ligament in the cervical spine with human leukocyte antigen haplotype. Spine 16: 1249-1252, 1991

71. Saunders RL, Pikus HJ, Ball P: Four-level cervical corpectomy. Spine 23:2455-2461, 1998

72. Sodeyama T, Goto S, Mochizuki M, et al: Effect of decompression enlargement laminoplasty for posterior shifting of the spinal cord. Spine 24:1527-1532, 1999

73. Takatsu T, Ishida Y, Suzuki K, et al: Radiological study of cervical ossification of the posterior longitudinal ligament. J Spinal Disord 12:271-273, 1999

74. Terayama K: Genetic studies on ossification of the posterior longitudinal ligament of the spine. Spine 14:1184-1191, 1989

75. Vaccaro AR, Falatyn SP, Scuderi GJ, et al: Early failure of long segment anterior cervical plate fixation. J Spinal Disord 11: 410-415, 1998

76. Wellman BJ, Follett KA, Traynelis VC: Complications of posterior articular mass plate fixation of the subaxial cervical spine in 43 consecutive patients. Spine 23:193-200, 1998

77. Yamaura I, Kurosa Y, Matuoka T, et al: Anterior floating method for cervical myelopathy caused by ossification of the posterior longitudinal ligament. Clin Orthop 359:27-34, 1999

78. Yonemori K, Imamura T, Ishidou Y, et al: Bone morphogenetic protein receptors and activin receptors are highly expressed in ossified ligament tissues of patients with ossification of the posterior longitudinal ligament. Am J Pathol 150:1335-1347, 1997

79. Yonenobu I, Abumi K, Nagatra K, Tet al: Inter- and intraobserve reliability of the Japanese Orthopaedic Association Scoring System for evaluation of cervical compression myelopathy. Spine (In press)

80. Yonenobu K, Hosono N, Iwasaki M, et al: Laminoplasty versus subtotal corpectomy. A comparative study of results in multisegmental cervical spondylotic myelopathy. Spine 17: 1281-1284, 1992

81. Yonenobu K, Hosono N, Iwasaki M, et al: Neurologic complications of surgery for cervical compression myelopathy. Spine 16:1277-1282, 1991

Manuscript received March 29, 2002.

Accepted in final form May 21, 2002.

Address reprint requests to: Nancy Epstein, M.D., Long Island Neurosurgical Associates, 410 Lakeville Road, Suite 204, New Hyde Park, New York 11042. email: DCH3@ columbia.edu. 